\title{
Subsea drilling and wellhead load monitoring systems in the North Sea: A case study using a well access management system (WAMS)
}

\author{
Alireza Sadeghi ${ }^{*}$, Per Thomas Moe ${ }^{2}$, Alexander Hilley ${ }^{1}$ \\ ${ }^{1 *}$ Subsea Services, Structural Analysis Engineering, TechnipFMC, Norway \\ ${ }^{2}$ Subsea Services, Structural Analysis Engineering, TechnipFMC / Department of Science and Industrial Systems, University of South-Eastern Norway, \\ Norway
}

ARTICLE INFO

ARTICLE HISTORY:

Received: 15 January 2021

Revised: 19 January 2021

Accepted: 29 January 2021

Published: 31 January 2021

\section{KEYWORDS:}

Load monitoring system, drilling, subsea wellhead system, fatigue management, well access management system

\begin{abstract}
A B S T R A C T
In the last decade, the monitoring systems have become a crucial component for the drilling and oil wellhead operations. The importance of such systems has been magnified by oil companies and the regulatory bodies after the Gulf of Mexico incident on Deepwater Horizon rig that took place on $20^{\text {th }}$ of April 2010 in which eleven crewmen were killed. The data obtained by the monitoring systems can provide significant advisory data to assist the operators to mitigate the various risks during the operations and to reduce the maintenance cost of equipment. In this paper, an overview of recent studies on the existing monitoring systems for the drilling and oil wellhead operations technical challenges faced by operators and the benefits of monitoring systems has been presented. Furthermore, a case study of a recent monitoring system installed on a drilling rig in the North Sea has been reported. In the case study for the deployment of the Well Access Management System (WAMS), hardware architecture, qualification, design-based parameters for hardware selection and its calibration have been described and discussed. Some practical recommendations to improve the system have been listed, based on the lessons learned from engineering, design, commissioning and system behaviour during operation has been presented. High performance, hardware architecture modularity, minimal manpower interventions, maintenance cost reduction and extension of the wellhead life span are advantages of the system.
\end{abstract}

\section{INTRODUCTION}

During the last decade, the major oil and gas companies have made more widespread use of monitoring systems for structural integrity to reduce cost and to satisfy increased regulation. An example of regulator body requirement is the Bureau of Safety and Environmental Enforcement (BSEE) introducing guidelines for Real-Time Monitoring of Offshore Oil and Gas Operations in 2015.

During drilling, intervention and completion operations riser systems are exposed to high cyclic loads and as a consequence mechanical fatigue. The gradual degradation of the subsea equipment should be monitored in order to optimize maintenance and to prevent major incidents causing a delay in the production or environmental impact.

Monitoring systems measure actual fatigue exposure of the equipment and provide the operator with real-time data which in effect could increase the lifespan of the workover risers by providing advisory input to the operator and assist in maintenance planning.
In this paper, an overview of the recent studies on the existing monitoring systems for drilling and intervention operations, technical challenges faced by operators and benefits of monitoring systems had been performed. Furthermore, a case study of a TechnipFMC monitoring system installed on a recent rig in the North Sea has been presented. In the case study for the deployment of the Well Access Management System (WAMS), hardware architecture, qualification, design-based parameters for hardware selection and its calibration have been described and discussed. As a result of the study, some practical recommendations to improve the system have been listed, based on the lessons learned from engineering, design, commissioning and system behaviour during operation has been presented. 


\section{LITERATURE REVIEW}

The application of monitoring systems for subsea drilling and wellhead load is linked to broad topics, such as the structural integrity assessment, methodology, hardware, software infrastructure, cost, regulatory and compliance aspects. In this section, some of the noteworthy existing literature is presented.

One key challenge for offshore drilling operations is the estimation and migration of the fatigue life of wellheads. The wellhead is the uppermost part of the oil and gas well, and it may be exposed to very high cyclic loads during drilling and intervention. Prior to the 2000s wellhead design considered static forces only (mainly pressure). However, advanced finite element models of riser systems have demonstrated that for operations on shallow water fatigue is a major issue. Since then, significant efforts have been made to enhance wellhead designs with respect to fatigue and to more accurately assess material resistance to fatigue. Furthermore, a host of methods for reducing wellhead loads have also been implemented. Monitoring systems play an important role, however, since they potentially produce more accurate estimates of fatigue than models. The estimation of fatigue by numerical analysis is conservative since the true loads on the wellhead are not known (Jensen, 2013).

According to McNeill et al. (2018), subsea wellheads and drilling risers are subjected to high fatigue damage rates when vortex induced vibration (VIV) occurs due to high currents during offshore drilling activities. He noted that employing monitoring systems on the riser and BOP stack provides a direct window into the response of the system, circumventing the complexity of VIV analysis due to the conversion of the currents to riser/wellhead vibrations, as a result of the complex fluid-structure interaction.

Howells et al. (2015) discussed design challenges faced in extending the service life of older wells to improve the calculated fatigue lives and have submitted further steps like as in-service monitoring may be employed to achieve the fitness-for-purpose.

Wang et al. (2018) summarized the developments in the field of monitoring of offshore structures, outlining the typical monitoring scopes and related sensor technologies. The work also includes the design aspects and applications of monitoring systems for offshore structures.

Ge et al. (2014) recent efforts were performed to develop a new fatigue monitoring methodology for drilling riser systems due to both wave and vortex-induced-vibration (VIV) damages. To validate their methodology measurement and theoretical approach have been used.

Zhamangarin (2014) has illustrated the application methodology of the riser monitoring system for an open sea workover system, based on a case study.
Another benefit of deploying a measured approach for the monitoring system is the data acquired which can be deployed for various artificial neural networks (ANN) algorithms. In their studies, Wiebe and Taylor (2019) developed a product ready ANN algorithm based on a real system. They highlighted that the conventional fatigue design/life extension methodology is considered best practice but is computationally intense and requires considerable manual input.

Simpson and Lima (2019) reviewed the main driving forces during the riser design process and presented the evolution of riser technology in a range of different regions and described the influencing factors behind each development and the propagation of technology around the world. They presented a break down for the global trends in riser by type, size, water depth and market dominance by applying graphical visualizations of an extensive dataset of installed riser systems.

Enuganti (2016) has studied the success factors of deepwater riser monitoring systems by applying a case study from Chevron-SCR monitoring system project. As their lessons learned for the delivery, he mentioned the value added by customer engagement from concept design through delivery, the importance of redundancy of the cables on the seabed as they were prone to damage. Furthermore, installation complexity was identified as a major challenge which had to be tackled in stages for a complex online monitoring system.

Transportation Research Board 2015 Executive Committee (2015) in their workshop report discussed the technical, regulatory and challenges of real-time monitoring (RTM). The study concluded that there are financial returns using RTM technology and, in the discussion, they have deliberated that due to rapid evolvement of data flow and information technology, it shall be taken into consideration that today's top-end solutions are likely to be obsolete within a 5-year segment. In their recommendations for the regulatory intervention, they highlighted that drilling highrisk wells will be the starting point of the gradual government regulations to use RTM.

\section{CHALLENGES DURING THE DRILLING OPERATION}

Table 1 provides an overview of the most common challenges faced by the operators during drilling operations observed in this study. 
Table 1. Challenges during the drilling operation Most common operators' challenges

To be compliant with the expanding monitoring requirement set by different regulatory bodies.

To screen the flex joint angle when running tools in real-time.

To have an unsophisticated solution for possible loss of well integrity during the drilling.

Need for sensor raw data from the previous operations or the upcoming operations with similar attributes.

Need for a measurement approach versus theoretical approach from the sensor data accrued during the operation.

Need for a real-time advisory monitoring system for critical operational decision-making situations such as the structural integrity of the stack in correlation to the meteorological conditions.

Need for a centralized solution to store the historical loading data of the wellhead accrued by a measurement approach.

\section{BENEFITS OF SUBSEA DRILLING AND WELLHEAD LOAD MONITORING SYSTEMS}

A summary of the most common benefits of utilizing drilling and wellhead load monitoring systems for operators observed in this study is presented in Table 2 .

Table 2. Benefits of drilling and load monitoring systems

\begin{tabular}{|c|}
\hline \\
\hline 1, \\
\hline \\
\hline \\
\hline $\begin{array}{l}\text { Collects raw data which enables prediction analysis. Predictive } \\
\text { models can assess the expected load on wellhead from the } \\
\text { upcoming drilling operations based on various defined } \\
\text { conditions. }\end{array}$ \\
\hline ice life by tracking historical wellhead fatigue \\
\hline Enables the reusability of critical wells \\
\hline \\
\hline
\end{tabular}

\section{FATIGUE AND DAMAGE CONCEPTS: A MONITORING SYSTEM APPLICATION}

\subsection{Fatigue}

Fatigue failure of structural members subjected to repeating cyclic loading occurs due to gradually accumulated damage to the materials of the structure. This cumulative phenomenon is typically an irreversible and an arbitrary energy dissipation process.
Currently, the Miner-Palmgren's linear fatigue cumulative damage formula is broadly employed. The Miner-Palmgren's formula was defined applying the stresslife curves and stress-number (SN) curves to determine the failure of the structures.

Eurocode (2009) expresses the actual damage that occurs in the structure related to the design fatigue life (Olsson \& Peterson, 2010; Amaravel \& Apparao, 2015). The fatigue formulas by DNV (2015) and API (2019) are commonly used in the offshore industry. The accumulated fatigue is of particular interest for the operators. Monitoring systems can facilitate a measured approach utilizing the various formulas or by machine learning algorithms.

Further verification can be implemented by comparing the results obtained from the monitoring systems (direct measured approach) to the FEA outputs or a statistical method result. Example of a statistical method such as that proposed by Wormsen (2007) on non-local stress approach for fatigue assessment based on weakest-link theory and statistics of extremes can be mentioned.

\subsection{Damage index}

The extent of damage of structural members under monotonic, cyclic or fatigue loading can be identified and quantified by a factor known as damage index (DI). Damage indices are based on different characteristics such as the number of cycles, stiffness, ductility and energy. Examples of such indices are DI of Park-Ang (1985) and SadeghiNouban (2016). It is worth noting that formulas like the one proposed by Sadeghi and Nouban (2016) are based on an energetic analysis method where the required data can be found either from the numerical simulation of structures or from the field data. Such formulas can be adapted by utilization of monitoring systems via the measured data from the sensors.

\section{WAMS A TECHNIPFMC PRODUCT}

\subsection{Overview}

TechnipFMC is a global oil and gas company that provides complete project life cycle services for the energy industry. Company products and systems are continuously evolving and expanding to increase efficiency and customer satisfaction.

TechnipFMC offers WAMS for structural monitoring of riser systems and wells. A Structural Analysis Engineering team performs analysis for the design of wellhead and riser systems and delivers analysis and monitoring services for offshore campaigns covering exploration, development, abandonment and well integrity scopes for both shallow water and deep-water campaigns. 
Typical scope of work for the analysis of wellheads, conductors and marine risers includes but is not limited to the following:

- Analysis of drilling, completion and workover riser systems,

- Confirm the acceptability of the riser, wellhead and conductor response to ensure the integrity of assets.

- Assessment undertaken for marine riser and workover operations with the subsea blowout preventer (BOP).

As an inhouse foundation block for the integrated analysis and measurement services provided, WAMS has been introduced as the monitoring system.

WAMS is a flexible and configurable system for monitoring and reporting structural integrity, riser and wellhead system. WAMS enables load monitoring for a given system, where the purpose of load monitoring among others is to:

- Document load histories and fatigue damage accumulation components, during all normal, extreme and accidental events

- Calculate fatigue damage accumulation for critical riser components, during riser deployment and recovery,

- provide input for inspection and maintenance routines.

\subsection{Hardware architecture}

WAMS has configurable architecture adjustable to the need of a particular riser and wellhead system. The system configuration may depend on parameters such as power mode during operation, data retrieval methods, fatigue and load calculation needs and accessibility.

Table 3. Required parameters for WAMS hardware selection

\begin{tabular}{|l|l|}
\hline System mode & (Local), (Live/real-time) \\
\hline Water depth & $(<500 \mathrm{~m}),(500-1500 \mathrm{~m}),(1500-3000 \mathrm{~m})$ \\
\hline $\begin{array}{l}\text { Sensor } \\
\text { Accuracy }\end{array}$ & (High), (Standard) \\
\hline $\begin{array}{l}\text { Primary } \\
\text { WAMS } \\
\text { power source }\end{array}$ & (Share power), (Umbilical), (Battery) \\
\hline $\begin{array}{l}\text { Subsea Data } \\
\text { Transmission }\end{array}$ & (Umbilical), (Acoustic), (Optical), (ROV) \\
\hline $\begin{array}{l}\text { HMI Display } \\
\text { Terminal }\end{array}$ & (No terminal), (Tablet), (Monitor) (Well \\
\hline $\begin{array}{l}\text { Topside HW } \\
\text { certification }\end{array}$ & (Atex), (UL) \\
\hline
\end{tabular}

WAMS parts are selected during the start of the project from the WAMS pool and the part selection is performed by WAMS system engineer base on fitness for purpose. The locations to be monitored are based on output from the preliminary riser analysis and experience from prior systems. The sensor positions are selected to collect information about the locations of the riser system most exposed to high static and cyclic loads, typical locations with small areal cross-sections that are exposed to high tensional loads or bending moments. Table 3 illustrates the required parameters for WAMS hardware selection. Figs. 2 and 3 shows the setup and the WAMS HW location on the $\mathrm{BOP}$ of a recent rig.

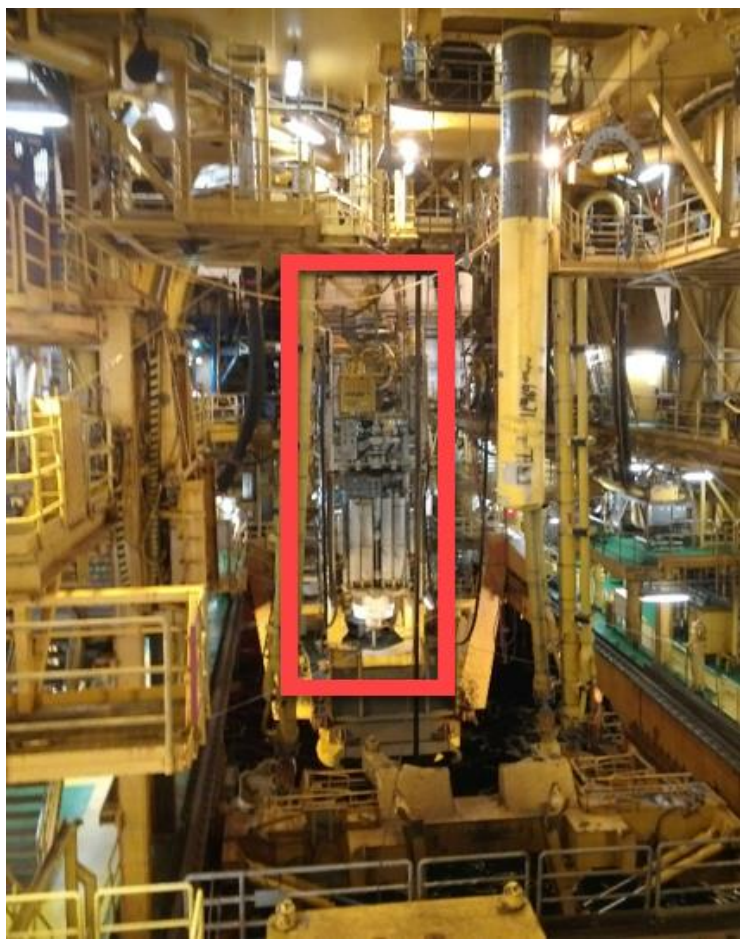

Fig. 1. WAMS setup installation area

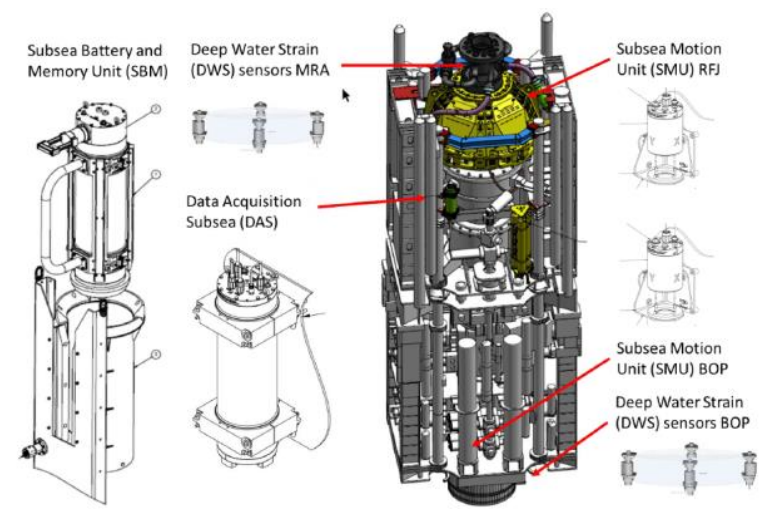

Fig. 2. WAMS hardware selection (Sønåsen, 2020)

\subsection{Qualification}

For the product to be able to be used in operation, the WAMS hardware has been through qualification according to the API $17 \mathrm{~F}$ standard. a qualification program has been established to define functional, environmental, and system tests with corresponding acceptance criteria.

Qualification tests were performed to confirm the performance of the equipment at its specified design 
conditions and its compatibility with the environment.

In the qualification effort, apart from the standard specified, the core has been through performance test and calibration to validate the results from the respective part.

\subsection{Calibration}

Calibration is required at both sensor and system levels in order to ensure the accuracy of fatigue estimates. A common definition of sensor and system performance parameters is important. In the academic and commercial literature to little emphasis is usually placed on describing how calibration tests have been performed and on the documentation of performance. In the case of WAMS definitions of the trueness and precision of sensor measurement have been adopted from ISO 5725-1:1994 (1994). Testing at sensor level is performed according to ASTM E 74-18e1 (2018).

Sensors for completion and workover systems are calibrated regularly in the workshop by applying known loads and displacements. Sensor systems on drilling risers are regularly calibrated offshore as it due to the size and continuous use of the equipment cannot be performed in a workshop environment. The offset of strain sensors should be performed in a state of zero or otherwise known loads. The offset of inclinometers is performed onshore. For the accurate measurement of bending moments and mechanical fatigue, however, sensor gain (tension/bending moment vs sensor output) is more important. The estimation of gain is performed using pressure and bending tests. Validation is also performed by applying known loads subsea in a socalled box test. In this test, the drilling rig is moved in a box pattern and exposes the sensors to known loads. With the analysis of the data obtained from test results, the system is initialization and is ready for use. Parameters required for each test depends on the stack to be monitored.

\section{DISCUSSION}

Based on the study performed from the literature review, the lessons learned from WAMS's engineering, design, commissioning and system behaviour during operation the following shall be mentioned:

- A monitoring system has to be deployed based on 5year design life with yearly recertification and end of lifecycle system migration plan.

- In the design of the monitoring system, the architectures have to be developed in three modules of software, hardware and methodology to provide the benefit of upgrading individual module separately.

- Process improvement is needed for installation, calibration and modification of the monitoring systems to reduce the related cost.
- Monitoring system solutions are currently optimized to be autonomous for a period up to a year. However, in the future fatigue monitoring systems are likely to be more integrated with the riser system design.

- Yearly review of system compliance with the cybersecurity protocols and guidelines.

- Continuous development of algorithms is required to provide analytic data to detect and manage structural failures in the wellhead, and conductor instability issues.

- Further study on the possibility to utilize additive manufacturing for customized hardware such as brackets and clamps.

\section{CONCLUSIONS}

In this paper, an overview of the current studies on the existing monitoring systems for the drilling and oil wellhead operations, the technical challenges faced by operators and the benefits of monitoring systems had been performed. Furthermore, a case study of a recent TechnipFMC monitoring system installed on a rig in the North Sea has been outlined. In the case study for the deployment of the Well Access Management System (WAMS), hardware architecture, qualification, design-based parameters for hardware selection and its calibration have been described and discussed.

As a result of the study base on the lessons learned from WAMS engineering, design, commissioning and system behaviour during operation has been discussed and recommendations were given. From the case study, the advantages of WAMS due to its, high performance, hardware architecture modularity, minimal manpower interventions, maintenance cost reduction and extension of the wellhead life span has been observed.

As a conclusion, the monitoring system is gradually becoming a permanent component of each operation and with the advancement of the inline technologies, it can be expected that monitoring systems on the drillship or semisubmersible rig will have following features:

- All floating vessels will be required to have a monitoring system onboard,

- Able to remotely perform the system calibration,

- Monitoring sensors will be plug and play,

- The data centres will collect data from different operations to provide enhanced advisory services,

- Monitoring system software architecture will be digitalized with cloud platforms,

- Additive manufacturing will be utilized for the customized hardware to reduce to lead time of monitoring systems. 


\section{CONFLICT OF INTEREST STATEMENT}

The authors declare that there is no conflict of interest.

\section{REFERENCES}

[1] Amaravel R., Apparao G. (2015), Studies on various theories and models for assessing the remaining life of damaged railway bridges-review (Fatigue and fracture mechanics approach), International Research Journal of Engineering and Technology, 2(5), 183-195.

[2] American Petroleum Institute (2019), Planning, Designing, and Constructing Fixed Offshore Platforms - Load and Resistance Factor Design (API RP 2ALRFD).

[3] American Society for Testing and Materials (2018), Practices for Calibration and Verification for ForceMeasuring Instruments (ASTM E 74-18e1). http://doi:10.1520/e0074-18e01

[4] Det Norske Veritas Germanischer Lloyd (2015). Design of offshore steel structures, general - LRFD method (DNVGL-OS-C101).

[5] European standard (2009), Eurocode 3: Design of steel structures-Part 1-9: Fatigue (EN 1993-19:2005/AC:2009).

[6] Enuganti, P. (2016). Ensuring Success of Deepwater Riser Monitoring Systems. Advances in Riser \& Flexibles Design and Technology. Offshore Pipeline Technology, Amsterdam, Netherlands, February 2016, $1-30$.

[7] Ge, M. L., Kannala, J., Li, S., Maheshwari, H., \& Campbell, M. (2014). A new riser fatigue monitoring methodology based on measured accelerations. 33rd International Conference on Ocean, Offshore and Arctic Engineering OMAE2014. American Society of Mechanical Engineers (ASME), San Francisco, California, USA, June 2014, 1-7.

[8] Howells, H., Rimmer, A., \& Ward P. (2015). Subsea Well Life Extension Fatigue Challenges. SPE Bergen, Society of Petroleum engineers, Bergen - Norway, 1 5.

[9] International Organization for Standardization (1994), ISO 5725-1:1994 Accuracy (trueness and precision) of measurement methods and results - Part 1: General principles and definitions (ISO 5725-1:1994).

[10] Jensen, G. A. (2013). Riser and wellhead monitoring for improved offshore drilling operations, CeSOS Highlights and AMOS Visions Conference, NTNU, Trondheim. http://www.cesos.ntnu.no/attachments/078_S2_Gullik \%20Jensen.pdf

[11] McNeill, S., Kluk, D., \& Bhalla, K. (2018). Subsea monitoring data improves riser-wellhead system design and operation. Offshore magazine. https://www.offshore-

mag.com/subsea/article/16762236/subsea-monitoringdata-improves-riserwellhead-system-design-andoperation

[12] Olsson K., Peterson J. (2010), Fatigue assessment methods for reinforced concrete bridges in Euro Code, Chalmers University of Technology, Goteborg, Sweden.

[13] Park Y. J., Ang A. H. S. (1985), Mechanistic seismic damage model for reinforced concrete, Journal of Structural Division (ASCE), 111(4), 722-739.

[14] Sadeghi, Kabir, \& Nouban, Fatemeh. (2016). Damage and fatigue quantification of RC structures. Structural Engineering and Mechanics, 58(6), 1021-1044. https://doi.org/10.12989/SEM.2016.58.6.1021

[15] Simpson, P., Lima, A. (2019). Deepwater Risers Historical Review and Future Projections. OTC Brasil, Offshore Technology Conference. Rio de Janeiro, Brazil, October 2019, 1-15.

[16] Sønåsen, K. O., Moe, P. T., Hansen, M., Fjeldstad, D. A., Gustad, H., Sadeghi, A., \& Hilley, A. (2020) [Unpublished Manuscript]. Significant Reduction of Well System Fatigue By Use Of Reactive Flex Joint With Integrated Monitoring System. Manuscript submitted for publication.

[17] Transportation Research Board 2015 Executive Committee (2015). Application of Real-Time Monitoring of Offshore Oil and Gas OperationsWorkshop Report. Transportation Research Board, Houston, Texas, USA, April 2015, 1-70.

[18] Wang, P., Tian, X., Peng, T., \& Luo, Y. (2018). A review of the state-of-the-art developments in the field monitoring of offshore structures. Ocean Engineering. 147, 148-164. https://doi.org/10.1016/j.oceaneng.2017.10.014

[19] Wiebe, C., Taylor, B. (2019). Flexible Life Extension with Innovative Monitoring. AOG 2019. Australasian Oil \& Gas Exhibition \& Conference (AOG). Perth, Australia, March 2019, 1-18.

[20] Wormsen, A., Sjödin, B., Härkegård, G., \& Fjeldstad, A. (2007). Non-local stress approach for fatigue assessment based on weakest-link theory and statistics of extremes. Fatigue \& Fracture of Engineering Materials and Structures, 30(12), 1214-1227. https://doi.org/10.1111/j.1460-2695.2007.01190.x

[21] Zhamangarin, R. (2014). Condition Based Maintenance for Well Access Systems. Master Dissertation, University of Stavanger. UiS Brage. https://uis.brage.unit.no/uisxmlui/bitstream/handle/11250/273925/Zhamangarin\% 2C\%20Rauan.pdf?sequence $=1 \&$ is Allowed $=\mathrm{y}$ 